\title{
Tetrabromobisphenol A Induces MMP-9 Expression via NADPH Oxidase and the activation of ROS, MAPK, and Akt Pathways in Human Breast Cancer MCF-7 Cells
}

\author{
Gi Ho Lee ${ }^{\dagger}$, Sun Woo Jin ${ }^{\dagger}$, Se Jong Kim, Thi Hoa Pham, Jae Ho Choi and Hye Gwang Jeong \\ Department of Toxicology, College of Pharmacy, Chungnam National University, Daejeon, Korea
}

\begin{abstract}
Tetrabromobisphenol A (TBBPA), the most common industrial brominated flame retardant, acts as a cytotoxic, neurotoxic, and immunotoxicant, causing inflammation and tumors. However, the mechanism of TBBPA-induced matrix metalloproteinase-9 (MMP-9) expression in human breast cancer cells is not clear. In human breast cancer MCF-7 cells, treatment with TBBPA significantly induced the expression and promoter activity of MMP-9. Transient transfection with MMP-9 mutation promoter constructs verified that NF- $\mathrm{KB}$ and AP-1 response elements are responsible for the effects of TBBPA. Furthermore, TBBPA-induced MMP-9 expression was mediated by NF- $\kappa \mathrm{B}$ and AP-1 transcription activation as a result of the phosphorylation of the Akt and MAPK signaling pathways. Moreover, TBBPA-induced activation of Akt/MAPK pathways and MMP-9 expression were attenuated by a specific NADPH oxidase inhibitor, and the ROS scavenger. These results suggest that TBBPA can induce cancer cell metastasis by releasing MMP-9 via ROS-dependent MAPK, and Akt pathways in MCF-7 cells.
\end{abstract}

Key words: Tetrabromobisphenol A, MMP-9, ROS, MAPK, Akt

\section{INTRODUCTION}

Tetrabromobisphenol A (TBBPA) is the most important industrial brominated flame retardant (BFR) (1). BFRs are organobromine compounds that exert an inhibitory effect on combustion chemistry and tend to reduce the flammability of polymeric materials. BFRs are emitted through local sewage treatment systems that spread to homes, industries, or the environment, such that humans are readily exposed to BFRs in underwater environments. TBBPA and its derivatives have been detected in human blood samples and in eggs from predatory bird species $(2,3)$. Additionally, their presence in sediment and mussels indi-

Correspondence to: Hye Gwang Jeong, Department of Toxicology, College of Pharmacy, Chungnam National University, Daejeon 34134, Korea

E-mail: hgjeong@cnu.ac.kr

${ }^{\dagger}$ The first two authors contributed equally to this work.

This is an Open-Access article distributed under the terms of the Creative Commons Attribution Non-Commercial License (http:// creativecommons.org/licenses/by-nc/3.0) which permits unrestricted non-commercial use, distribution, and reproduction in any medium, provided the original work is properly cited. cates that TBBPA may impact on aquatic organisms $(4,5)$. Previous studies on the toxicity of BFRs have mainly focused on polybrominated diphenyl ethers $(3,6)$. However, previous studies reported that TBBPA plays role as a cytotoxicant, neurotoxicant, and thyroid hormone agonist, and shows weak estrogenic activity in mammalian cells $(3,7)$. Moreover, TBBPA was recently reported for its immunotoxic potential $(8,9)$.

The regulation of matrix metalloproteinase-9 (MMP-9) plays a key role in the inflammatory response, angiogenesis, wound healing, and the differentiation of human embryonic stem cells $(10,11)$. However, high expression of MMP-9 increases tumorigenesis and metastasis $(12,13)$. In particular, an elevated level of MMP-9 induces the metastasis and invasion of breast cancer, leading to death in patients with breast cancer (14). Overexpression of MMP-9 has been implicated in breast cancer development, angiogenesis, invasion, and metastasis $(15,16)$. Accumulating evidence has shown the critical role of both mitogen-activated protein kinase (MAPK) and PI3-K/Akt pathways in the regulation of MMP-9 production $(17,18)$. Activated MAPKs and Akt induce transcription factors, including nuclear factor-kappaB $(\mathrm{NF}-\kappa \mathrm{B})$ and activator protein-1 (AP-1), which regulate MMP-9 expression by an 
interaction with transcription factor-binding sites in MMP promoters (19). These transcription factors also regulate the expression of various genes, which are involved in tumorigenesis (20).

Reactive oxygen species (ROS) have emerged as an important proinflammatory mediator in various inflammatory diseases. For the activation of MMP-9 by ROS, various intracellular signaling pathways have been identified $(21,22)$. The main sources of ROS in cells, beside the respiratory chain, are NADPH oxidases (NOX). NOX is an important cellular source of ROS production under many pathologic conditions involved in superoxide generation from NADPH (23). The physiological functions of NOX play various roles in cell signaling, gene expression regulation, cell growth, differentiation, and death (24). NOX-derived ROS have been reported to activate various signaling pathways, such as Akt and MAPK (25), and the activation of transcription factors, such as NF- $\kappa$ B and AP$1(26,27)$. However, scant information on the regulation of MMP-9 expression by TBBPA is available. In this study, we investigated the effect of TBBPA on the regulatory mechanism of MMP-9 expression in human breast cancer MCF-7 cells.

\section{MATERIALS AND METHODS}

Materials. The TBBPA (97\%) was obtained from Sigma-Aldrich (St. Louis, MO, USA). RPMI 1640, fetal bovine serum (FBS), and penicillin-streptomycin were obtained from Gibco BRL (Grand Island, NY, USA). Lipofectamine $2000^{\mathrm{TM}}$ was obtained from Life Technologies, Inc (Carlsbad, CA, USA). A dual-luciferase assay system was obtained from Promega (Madison, WI, USA). Inhibitors against LY294002, PD98059, SB203580, and SP600125 were obtained from Calbiochem (La Jolla, CA, USA). Primary antibodies against phospho-Akt, phosphoERK1/2, phospho-p38 MAPK, phospho-JNK1/2, ERK1/2, p38 MAPK, JNK1/2, and secondary antibodies against horseradish peroxidase (HRP)-linked anti-mouse or antirabbit IgG were obtained from Cell Signaling Technologies (Beverly, MA, USA). Primary antibodies against $\beta$ actin, Lamin B1, NF- $\kappa$ B p65, c-Fos, c-Jun, Akt, and PKC $\alpha$ were obtained from Santa Cruz Biotechnology (Santa Cruz, CA, USA). Polymerase chain reaction (PCR) oligonucleotide primers were custom synthesized by Bioneer Co. (Daejeon, Korea). Polyvinylidene fluoride (PVDF) membranes and enhanced chemiluminescence (ECL) system were obtained from Amersham Pharmacia Biotech (Piscataway, NJ, USA).

Cell cultures. The human breast carcinoma MCF-7 cell line was obtained from the American Type Culture Collection (Manassas, VA, USA). The MCF-7 cells were cultured in RPMI 1640 supplemented with 10\% FBS, 100 $\mu \mathrm{g} / \mathrm{mL}$ streptomycin, and $100 \mathrm{U} / \mathrm{mL}$ penicillin in a humidified atmosphere of $5 \% \mathrm{CO}_{2}$ at $37^{\circ} \mathrm{C}$. To avoid the interference of growth factors in serum, MCF-7 cells were serumstarved for $24 \mathrm{hr}$ and then stimulated with TBBPA.

Measurement of cell viability. Conventional MTT reduction and lactate dehydrogenase (LDH) leakage assays were used to determine MCF-7 cell viability following treatment with TBBPA. To measure cell viability, cells were seeded onto 48-well plates and treated with TBBPA (1$50 \mu \mathrm{M})$ for $24 \mathrm{hr}$. After incubation, MTT solution $(0.5$ $\mathrm{mg} / \mathrm{mL}$ ) was added to the 48 -well plates for $1 \mathrm{hr}$, and formazan crystals were solubilized with dimethylsulfoxide. The absorbance was measured at $550 \mathrm{~nm}$ in a BioTek Synergy HT microplate reader (BioTek Instruments, Winooski, VT, USA). The media were measured using an LDH kit (Cayman, Ann Arbor, MI, USA) at $490 \mathrm{~nm}$ on a BioTek Synergy HT microplate reader (BioTek Instruments).

RNA preparation and real-time PCR (qRT-PCR). Total RNA was isolated from untreated and TBBPA-treated MCF-7 cells with RNAiso-plus Reagent (Takara, Tokyo, Japan). RNA was converted to cDNA using a reverse transcription kit (Promega). qRT-PCR was performed as MMP9 gene expression analysis by monitoring increases in SYBR reporter dye fluorescence. Primer sequences were as follows: MMP-9 forward, 5'-GTCATCCAGTTTGGTGTCGC-3'; MMP-9 reverse, 5'-GGACCACAACTCGTCATCGT-3'; GAPDH forward, 5'-CCCTTCATTGACCTCAACTA-3' and GAPDH reverse, 5'-CCAAAGTTGTCATGGATGAC-3'. Expression values were normalized with GAPDH.

Transient transfection and luciferase activity assay. Promoter activity was determined using a dual-luciferase reporter assay system (Promega). MCF-7 cells were seeded onto 48-well plates overnight and transiently transfected with the MMP-9 promoter vector, the NF- $\mathrm{BB}$ or the AP-1 vector reporter vector, and the Renilla luciferase reporter vector using Lipofectamine. Renilla luciferase expression was used to normalized the luciferase activity.

Gelatin zymography. MCF-7 cells were seeded and incubated for $24 \mathrm{hr}$ and were then maintained in serumfree medium. After $24 \mathrm{hr}$ cell culture supernatants were collected, and loaded onto a $10 \%$ polyacrylamide gel containing $0.1 \%$ gelatin. Gels were washed with wash buffer (50 mM Tris- $\mathrm{HCl} \mathrm{pH} \mathrm{7.5,} \mathrm{and} \mathrm{2.5 \%} \mathrm{Triton} \mathrm{X-100)} \mathrm{and}$ incubated with zymography reaction buffer $(50 \mathrm{mM}$ Tris$\mathrm{HCl} \mathrm{pH} 7.5,1 \mathrm{mM} \mathrm{ZnCl} 2,5 \mathrm{mM} \mathrm{CaCl}_{2}, 150 \mathrm{mM} \mathrm{NaCl}$, and $40 \mathrm{mM} \mathrm{NaN}_{3}$ ) at $37^{\circ} \mathrm{C}$ for $24 \mathrm{hr}$. The gel was then stained with Coomassie brilliant blue in a solution of $45 \%$ methanol and 1\% acetic acid, and then destained for $1 \mathrm{hr}$. 
Area of MMP-9 activity appeared as transparent bands against the black background.

Westernblot analysis. After treatment, cell lysates were loaded onto sodium dodecyl sulfate polyacrylamide gel electrophoresis (SDS-PAGE). The gel was electrophoretically transferred to PVDF membranes, and blocked with 5\% skim milk. The PVDF membranes were incubated with overnight at $4{ }^{\circ} \mathrm{C}$ with primary antibodies and then incubated with HRP-conjugated anti-IgG secondary antibodies at room temperature for $2 \mathrm{hr}$. Membranes were then visualized using an ECL detection kit.

(A)

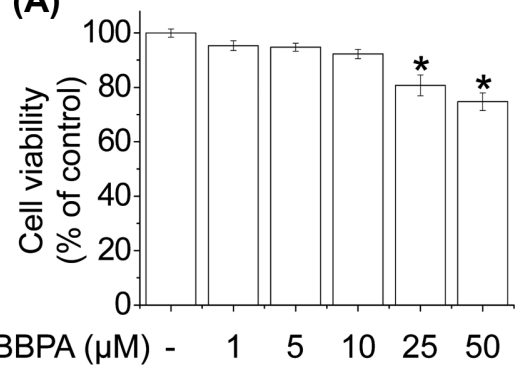

(C)

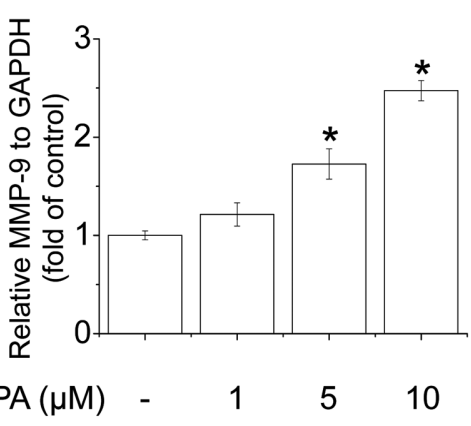

(E)

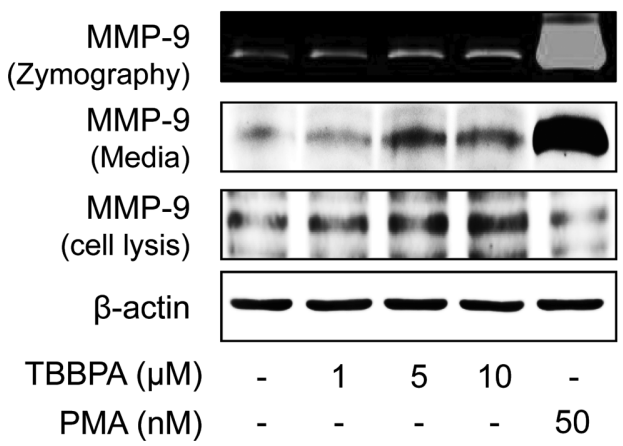

ROS production. The production of ROS by TBBPA in MCF-7 cells was using the redox-sensitive fluorescent dye $\mathrm{H}_{2}$ DCFDA. After treatment with TBBPA or vehicle, cells were probed with $2 \mu \mathrm{M} \mathrm{H}_{2} \mathrm{DCFDA}$ at $37^{\circ} \mathrm{C}$ for $20 \mathrm{~min}$ and washed with PBS. The fluorescence intensity was measured using a BioTek Synergy HT microplate reader (BioTek Instruments; excitation, $490 \mathrm{~nm}$; emission, $530 \mathrm{~nm}$ ).

Determination of NADPH oxidase activity. The activity of NADPH oxidase by TBBPA in MCF-7 cells was determined using the lucigenin chemiluminescence method. After treatment with TBBPA or vehicle for the

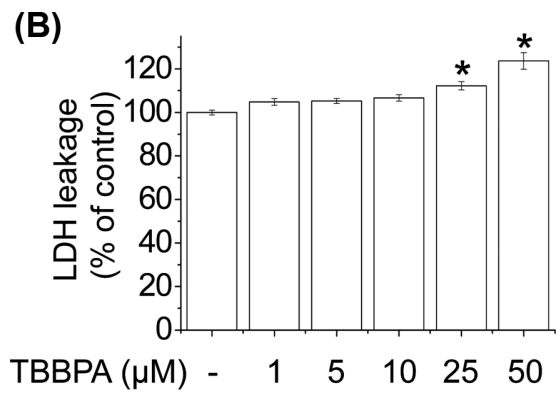

(D)

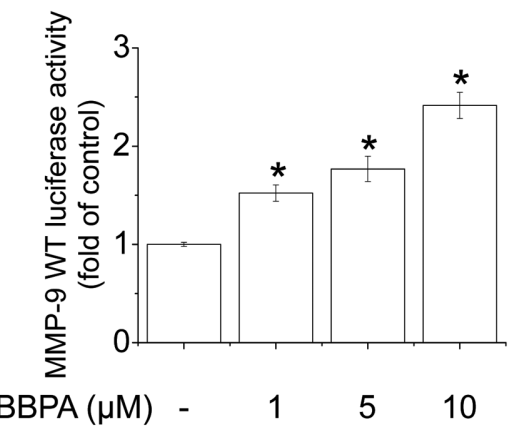

Fig. 1. Effects of TBBPA on MMP-9 expression and transcriptional activity. Cell viability was estimated by the (A) MTT and (B) LDH release assays. (C) Cells were treated with 1, 5, or $10 \mu \mathrm{M}$ TBBPA for $24 \mathrm{hr}$. Cells were then lysed and total RNA was prepared to analyze MMP-9 gene expression. (D) Cells were transfected with MMP-9-Luc for $4 \mathrm{hr}$ and then treated with 1, 5, or $10 \mu \mathrm{M}$ TBBPA for $24 \mathrm{hr}$. Cells were then harvested and assayed for luciferase activity. (E) Effect of TBBPA on MMP-9 activity levels in MCF-7 cells. Cells were treated with 1, 5, or $10 \mu \mathrm{M}$ TBBPA for $24 \mathrm{hr}$. MMP-9 activity in MCF-7 cells was determined by gelatin zymography and Western blot analysis. Each bar represents the mean \pm SD calculated from three independent experiments. *Significantly different from the control at $p<0.01$. 
indicated period of time, cells were gently scraped and centrifuged for $10 \mathrm{~min}$ at $4^{\circ} \mathrm{C}$. The cell pellets were then incubated with pre-warmed medium containing either $20 \mu \mathrm{M}$ lucigenin or $1 \mu \mathrm{M} \mathrm{NADPH}$, to initiate the reaction, followed by immediate measurement of chemiluminescence using a luminometer (Thermo Scientific, Waltham, MA, USA).

Statistical analysis. All data were demonstrated as means \pm SD from at least three representative tests. Statistical significance was assessed by one-way analysis of variance (ANOVA) followed by the Tukey-Kramer test. A value of $p<0.01$ was considered significantly different.

\section{RESULTS}

Induction of MMP-9 expression and activity by TBBPA in MCF-7 cells. We first measured the cytotoxicity of TBBPA by treating MCF-7 cells with the indicated concentrations of TBBPA for $24 \mathrm{hr}$ followed by the MTT and LDH assays. TBBPA (at concentrations ranging from
1 to $10 \mu \mathrm{M}$ ) had no cytotoxic effect on MCF-7 cells (Fig. 1A, 1B). To determine the effect of TBBPA on MMP-9 expression in MCF-7 cells, cells were treated with TBBPA at the indicated concentrations. As shown in Fig. 1C, MMP-9 mRNA expression was significantly increased by TBBPA treatment in a concentration-dependent manner. Next, the effect of TBBPA on MMP-9 promoter activity was examined. MCF-7 cells were transiently transfected with the luciferase reporter gene and treated with TBBPA for $24 \mathrm{hr}$. TBBPA significantly increased MMP-9 luciferase activity in a concentration-dependent manner (Fig. 1D). TBBPA also concentration-dependently increased MMP-9 protein expression, as well as enzyme activity in MCF-7 cells (Fig. 1E). These results indicate that TBBPA increases MMP-9 expression and activity in MCF-7 cells at nontoxic concentrations.

Involvement of Akt and MAPKs in TBBPA-induced MMP-9 expression. Several studies have reported that Akt and MAPKs are upstream modulators of NF- $\mathrm{kB}$ or AP-1, which regulates MMP-9 expression in cells $(10,28)$.

(A)

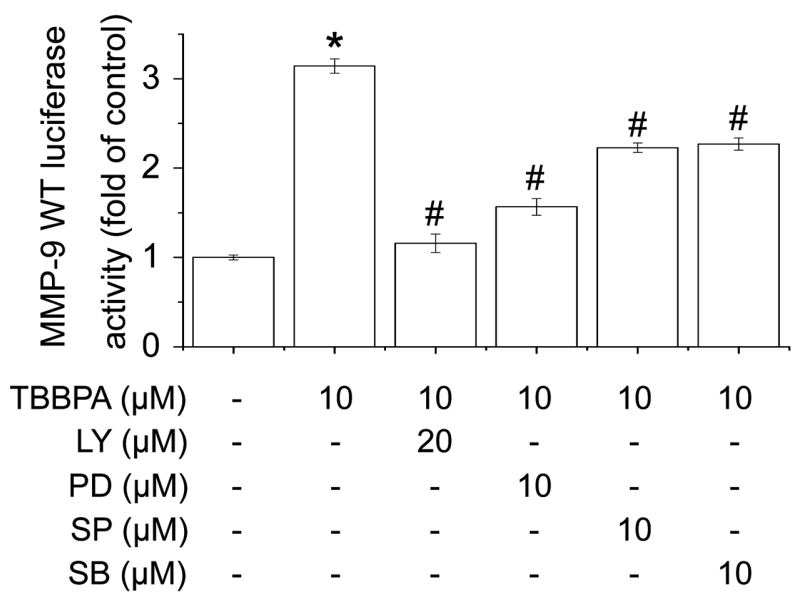

(B)

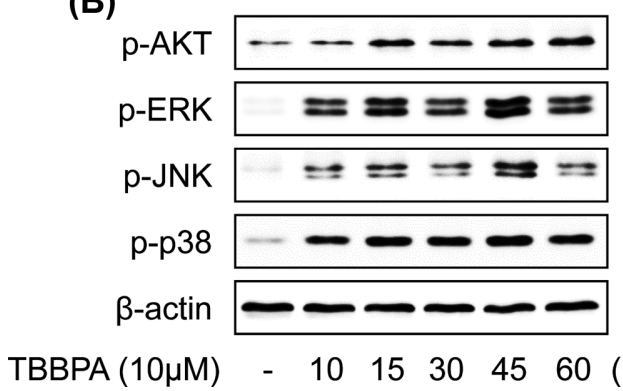

(C)

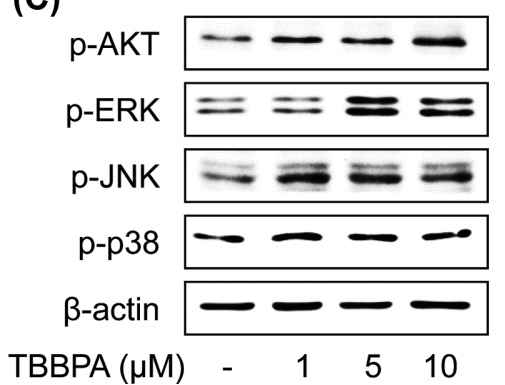

Fig. 2. Effect of TBBPA on the activation of Akt and MAPK signaling pathways in human breast cancer MCF-7 cells. (A) Cells were transfected with MMP-9-Luc and then pretreated with the indicated inhibitor for $60 \mathrm{~min}$ followed by $10 \mu \mathrm{M}$ TBBPA $24 \mathrm{hr}$. Cells were then harvested and assayed for luciferase activity. (B) Cells were treated with $10 \mu \mathrm{M}$ TBBPA for 10, 15, 30, 45, or 60 min or (C) 1, 5, or $10 \mu \mathrm{M}$ TBBPA for $30 \mathrm{~min}$, and the phosphorylation levels of Akt, ERK1/2, JNK, and p38 MAPK were measured by Western blot analysis. Each bar represents the mean \pm SD calculated from three independent experiments. *Significantly different from the control at $p<0.01$. "Significantly different from the TBBPA at $p<0.01$. 
We examined whether the activation of Akt, ERK1/2, JNK1/2, and/or p38 MAPK is involved in TBBPAincreased MMP-9 promoter activity using each upstream inhibitor (LY, PD, SP, and SB). MCF-7 cells were transiently transfected with the luciferase reporter gene, pretreated with the upstream inhibitor for $1 \mathrm{hr}$, and then stimulated with TBBPA for $24 \mathrm{hr}$. As shown in Fig. 2A, TBBPA-increased MMP-9 luciferase activity was suppressed by pretreatment with $\mathrm{LY}, \mathrm{PD}, \mathrm{SP}$, and SB in MCF-
7 cells. To examine these upstream pathways of NF- $\kappa B$ or AP-1, cells were treated with TBBPA for $1 \mathrm{hr}$, and then upstream proteins were analyzed by Western blot. The phosphorylation of Akt, ERK1/2, JNK1/2, and p38 MAPK increased significantly following TBBPA treatment in a concentration- and time-dependent manner (Fig. 2B, 2C). These results indicate that TBBPA-increased MMP-9 expression is mediated by the activation of Akt and MAPKs in MCF-7 cells.

(A)

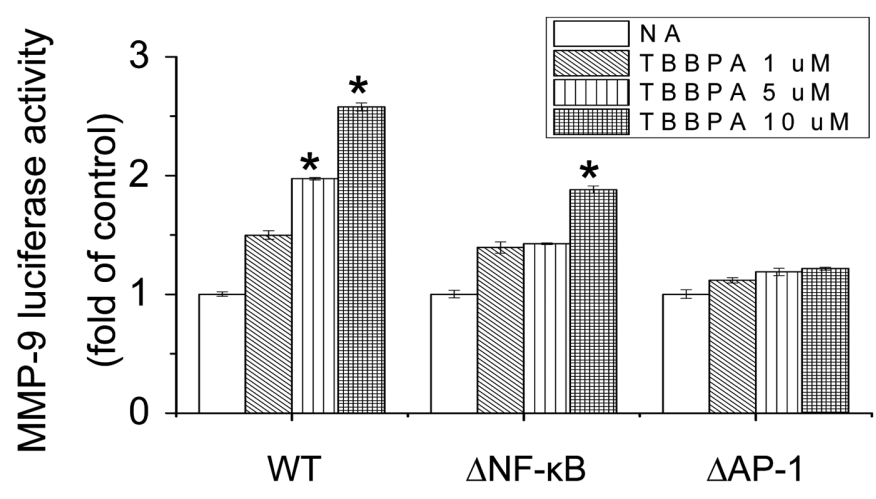

(B)

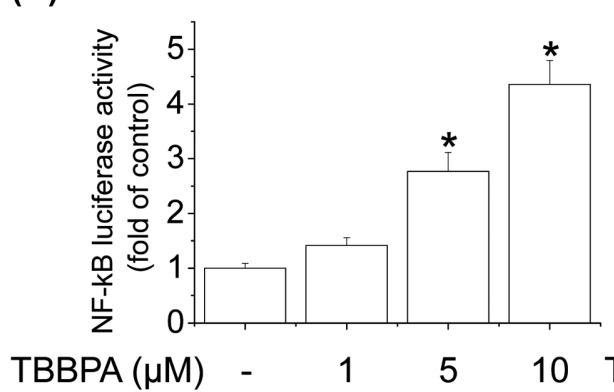

(C)

(D)

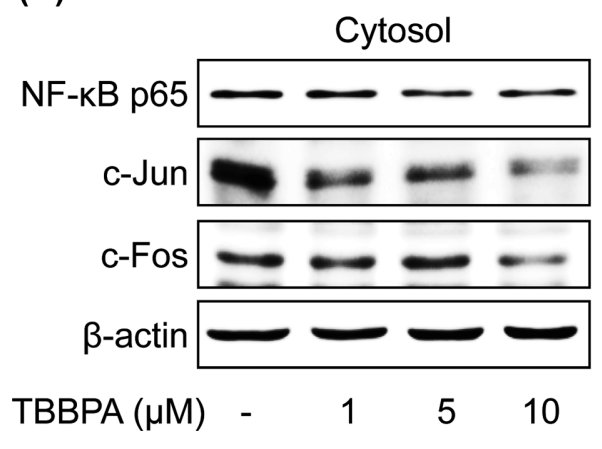

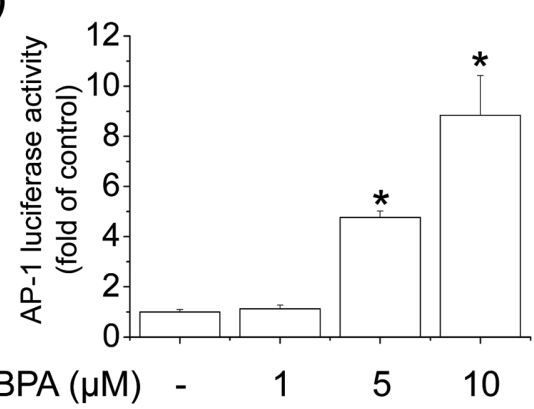

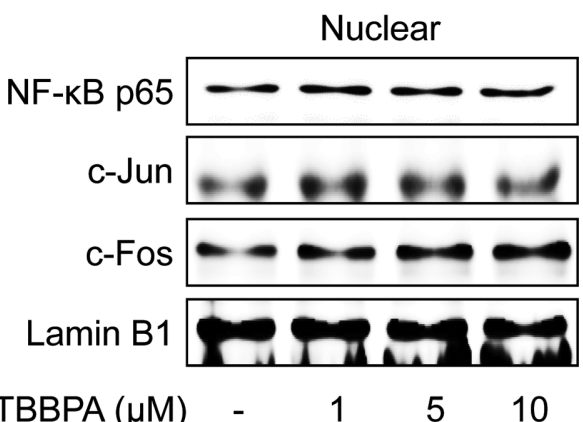

Fig. 3. Effect of TBBPA on the activity and expression of MMP-9 via NF- $\mathrm{B} B$ and AP-1 pathways in human breast cancer MCF-7 cells. (A) Mutations were introduced into the NF-KB- or AP-1-binding sites of pGL2-MMP-9WT. Cells were transfected with wild-type, $\Delta$ NF- $\mathrm{KB}$, or $\triangle \mathrm{AP}-1$ of MMP-9-Luc, and then treated with TBBPA (1-10 $\mu \mathrm{M})$ for $24 \mathrm{hr}$. Cells were then harvested and assayed for luciferase activity. $(B, C)$ Cells were transfected with NF- $\kappa B$ or AP-1-Luc and then treated with TBBPA $(1-10 \mu M)$ for 24 hr. Cells were harvested and assayed for luciferase activity. (D) Effects of TBBPA on NF-KB or AP-1 translocation. Cells were treated with TBBPA (1$10 \mu \mathrm{M})$ for $6 \mathrm{hr}$. Cytosolic or nuclear extracts were subjected to SDS-PAGE followed by Western blot analysis with anti-NF- $\mathrm{kB}$, anti-cJun, anti-c-Fos, and anti-Lamin B antibodies. Each bar represents the mean \pm SD calculated from three independent experiments. *Significantly different from the control at $p<0.01$. 
Activation of transcription factors NF- $K B$ and $A P-1$ in TBBPA-induced MMP-9 expression. NF- $\kappa \mathrm{B}$ and AP-1 are transcription factors of MMP-9 that play a central role in the regulation of MMP-9 mRNA expression in different cell types (29). To investigate which of these transcription factors regulates MMP-9 expression in MCF-7 cells, cells were transiently transfected with reporter genes that contain the wild-type MMP-9 promoter, an NF- $\kappa$ B sitemutated promoter, or an AP-1 site-mutated promoter. Cells were treated with TBBPA $(1,5$, or $10 \mu \mathrm{M})$, and MMP-9 transcriptional activity was analyzed according to luciferase activity. Treatment with TBBPA induced the transcriptional activity of the wild-type MMP-9 promoter, but had no effect on the site-mutated promoter of $\mathrm{NF}-\kappa \mathrm{B}$ or $\mathrm{AP}-1$
(A)

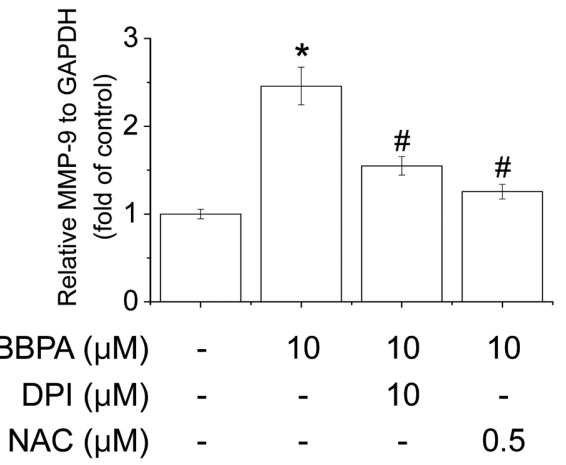

(B)

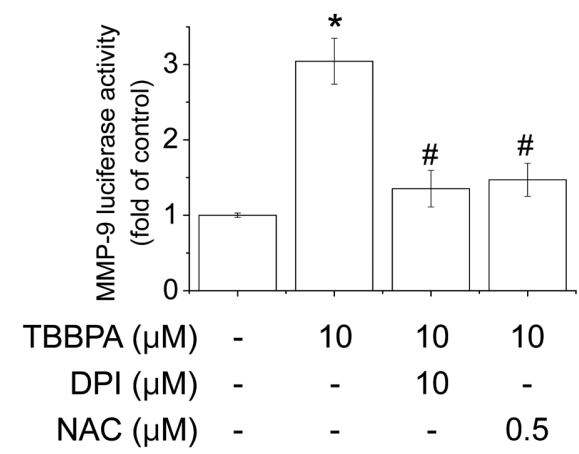

(E)

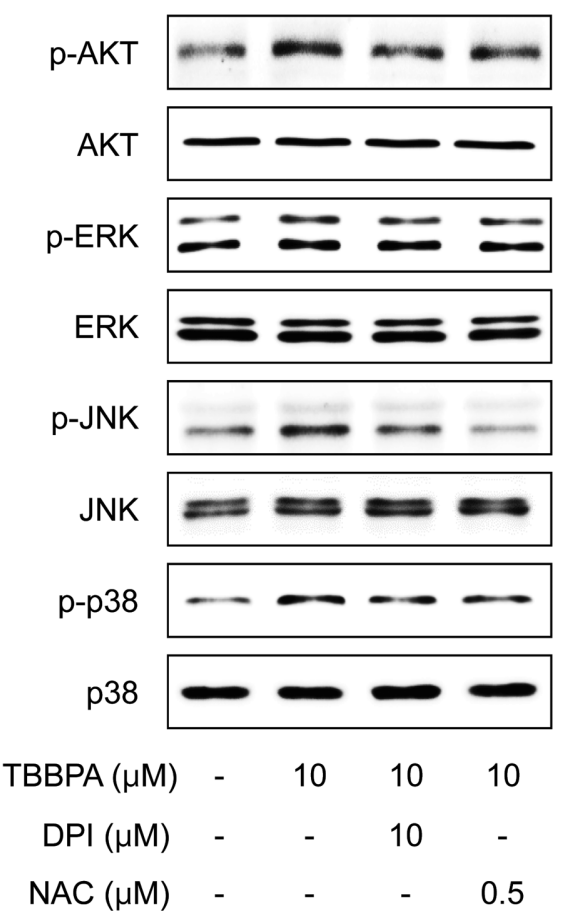

Fig. 4. Effects of NOX-derived ROS generation on TBBPA-induced MMP-9 expression. (A) Cells were treated with 1,5 , or $10 \mu M$ TBBPA. Cells were lysed and total RNA was prepared for MMP-9 analysis. (B) Cells were transfected with MMP9-Luc and then pretreated with inhibitors (NAC or DPI) for $30 \mathrm{~min}$ followed by TBBPA for $24 \mathrm{hr}$. Cells were then harvested and assayed for luciferase activity. (C) Cells were pre-treated with DPI for $30 \mathrm{~min}$ and TBBPA was added for an additional $10 \mathrm{~min}$. Cells were harvested to measure NADPH activity as described above. (D) Cells were pre-treated with NAC or DPI for $30 \mathrm{~min}$, and TBBPA was added for an additional $20 \mathrm{~min}$, followed by $\mathrm{H}_{2}$ DCFDA for $30 \mathrm{~min}$. Each bar represents the mean $\pm \mathrm{SD}$ calculated from three independent experiments. (E) Cells were pre-treated with NAC or DPI for $30 \mathrm{~min}$ and TBBPA was added. The extracts were analyzed for Akt and MAPK activation using antibodies against phosphorylated Akt, ERK1/2, JNK, and p38 MAPK by Western blot analysis. *Significantly different from the control at $p<0.01$. "Significantly different from the TBBPA at $p<0.01$. 
(Fig. 3A). To further determine the transcription factor involved in MMP-9 promoter activity induced by TBBPA, MCF-7 cells were transiently transfected with the respective reporter vector containing tandem repeats of the NF$\kappa \mathrm{B}-$ or AP-1-binding sites. TBBPA significantly increased NF- $\kappa \mathrm{B}$ - or AP-1-responsive luciferase activity in a concentration-dependent manner (Fig. 3B, 3C). To examine which of these transcription factors is involved in the activation of MMP-9 transcription by TBBPA, we examined the effect of TBBPA on the nuclear translocation of NF$\kappa \mathrm{B}$ p65 (a major subunit of NF- $\kappa \mathrm{B}$ ) or c-Jun and c-Fos (major subunits of AP-1), which are required for their respective transcriptional activities. The nuclear translocation of NF-kB p65, c-Jun, and c-Fos was significantly increased by TBBPA treatment in a concentration-dependent manner (Fig. 3D). These results demonstrate that TBBPA-stimulated MMP-9 induction is mediated by the transcriptional activation of both $\mathrm{NF}-\kappa \mathrm{B}$ and $\mathrm{AP}-1$ in MCF-7 cells.

NADPH oxidase-derived reactive oxygen species induced by TBBPA control the activation of Akt and MAPK signaling pathways. ROS have been demonstrated to induce the expression of inflammatory proteins in various cell types and stimulate Akt and MAPKs (25). The regulation of ROS activation is involved in NADPH oxidase (NOX) under various pathological conditions (30). Thus, we investigated whether TBBPA-induced MMP-9 expression is involved in NOX-dependent ROS generation. As shown in Fig. 4A, 4B, a NOX inhibitor (DPI) or an ROS scavenger (NAC) significantly suppressed TBBPAinduced MMP-9 gene expression and promoter activity. Additionally, TBBPA-induced NOX activity and ROS generation were inhibited by pre-treatment with NAC or DPI (Fig. 4C, 4D). These results indicate that NOX-dependent ROS generation plays a critical role in TBBPA-induced MMP-9 expression. Further, we determined whether TBBPA-induced phosphorylation of Akt and MAPKs was mediated through ROS generation. As shown in Fig. 4E, NAC or DPI significantly decreased the phosphorylation of Akt and MAPKs stimulated by TBBPA. These results indicate that NOX-derived ROS play a key role in TBBPAinduced MMP-9 expression in MCF-7 cells.

\section{DISCUSSION}

Our findings establish that TBBPA increases MMP-9 expression via NF- $\mathrm{KB}$ and AP-1 activation through ROSdependent Akt/MAPK signaling pathways in human breast cancer MCF-7 cells.

The activation of transcription factors, such as NF- $\kappa \mathrm{B}$ and AP-1, is a necessary step in MMP-9 expression, leading to remodeling of the extracellular matrix, as well as membrane degradation and the induction of angiogenesis
(19). In the present study, TBBPA increased the transcriptional activation of the MMP-9 promoter. Moreover, promoter mutation analysis indicated that the major targets of TBBPA were the binding sites of both NF- $\kappa$ B and AP-1. These results are consistent with our pervious study, in which TBBPA increased NF- $\kappa$ B- or AP-1-responsive luciferase activity in Raw 264.7 cells (31). NF- $\mathrm{KB}$ is a transcriptional activator of MMP-9 and is combined with inhibitory molecules, such as the IкB family, and are sequestered in the cytosol. Stimulation of cells via various signaling pathway triggers the phosphorylation of $\mathrm{I} \kappa \mathrm{B}$, leading to its proteasome-mediated degradation and translocation into the nuclei (32). AP-1 is a transcriptional activator of MMP-9 that is composed of a dimer of c-Jun and c-Fos protein families $(33,34)$. AP-1 is activated by modulation with other transcriptional regulators and is further regulated by upstream kinases, such as MAPKs, to induce various signal transduction pathways (35). In the present study, TBBPA increased the nuclear translocation of NF$\kappa \mathrm{B}$ p65, c-Jun, and c-Fos. These results indicate that TBBPA increases MMP-9 expression in MCF-7 cells by enhancing the activity of the transcription factors NF- $\kappa \mathrm{B}$ and AP-1. Several studies have shown that activation of $\mathrm{NF}-\kappa \mathrm{B}$ and AP-1 is triggered by Akt and MAPK signaling pathways (36). In the present study, TBBPA increased the phosphorylation of Akt, ERK1/2, JNK1/2, and p38 MAPK. Additionally, treatment with LY (Akt inhibitor), PD (ERK1/2 inhibitor), SP (JNK1/2 inhibitor), or SB (p38 inhibitor) reduced the TBBPA-increased promoter activity of MMP-9. These results suggest that TBBPA increases MMP-9 expression in MCF-7 cells by enhancing the activation of Akt and MAPKs.

Recent studies have reported that ROS generation can activate various signaling pathways, such as Akt and MAPK (25). Additionally, Akt and MAPK signaling pathways are activated in response to oxidant injury. Therefore, we further examined the mechanism of MMP-9 signal transduction by TBBPA-induced ROS generation in MCF-7 cells. In the present study, TBBPA-induced MMP-9 expression was attenuated by the NOX inhibitor, DPI, and the ROS scavenger, NAC. Additionally, our data also showed that the TBBPA-increased phosphorylation of Akt and MAPK was inhibited by treatment with DPI and NAC. These results suggest that ROS generation by TBBPAinduced NOX plays an important role in MMP-9 expression. However, whether TBBPA-induced ROS production is mediated by other sources remains an interesting topic to be addressed in future studies.

TBBPA is toxic to a variety of mammalian cell lines, as well as primary cells including cerebellar granule cells, splenocytes, and hepatocytes $(37,38)$. However, the effect of TBBPA on MMP-9 expression in breast cancer cells is unknown, which served as the basis for the present study. In conclusion, our study demonstrated that TBBPA induced 
MMP-9 expression via ROS-dependent Akt/MAPK signaling pathways, which was mediated via increased NF$\mathrm{\kappa B}$ and AP-1 activation in MCF-7 cells. Our findings indicate that TBBPA may affect cancer development, invasion, and metastasis in spite of the lack of toxicity data for TBBPA in MCF-7 cells.

\section{ACKNOWLEDGMENTS}

This work was supported by research fund of Chungnam National University.

\section{CONFLICT OF INTEREST}

None of the authors declares a conflict of interest.

Received October 31, 2018; Revised November 6, 2018; Accepted November 6, 2018

\section{REFERENCES}

1. de Wit, C.A. (2002) An overview of brominated flame retardants in the environment. Chemosphere, 46, 583-624.

2. Berger, U., Herzke, D. and Sandanger, T.M. (2004) Two trace analytical methods for determination of hydroxylated PCBs and other halogenated phenolic compounds in eggs from Norwegian birds of prey. Anal. Chem., 76, 441-452.

3. Birnbaum, L.S. and Staskal, D.F. (2004) Brominated flame retardants: cause for concern? Environ. Health Perspect., 112, 9-17.

4. Morris, S., Allchin, C.R., Zegers, B.N., Haftka, J.J., Boon, J.P., Belpaire, C., Leonards, P.E., Van Leeuwen, S.P. and De Boer, J. (2004) Distribution and fate of HBCD and TBBPA brominated flame retardants in North Sea estuaries and aquatic food webs. Environ. Sci. Technol., 38, 5497-5504.

5. Saint-Louis, R. and Pelletier, E. (2004) LC-ESI-MS-MS method for the analysis of tetrabromobisphenol $\mathrm{A}$ in sediment and sewage sludge. Analyst, 129, 724-730.

6. Darnerud, P.O. (2003) Toxic effects of brominated flame retardants in man and in wildlife. Environ. Int., 29, 841-853.

7. Kitamura, S., Suzuki, T., Sanoh, S., Kohta, R., Jinno, N., Sugihara, K., Yoshihara, S., Fujimoto, N., Watanabe, H. and Ohta, S. (2005) Comparative study of the endocrine-disrupting activity of bisphenol A and 19 related compounds. Toxicol. Sci., 84, 249-259.

8. Pullen, S., Boecker, R. and Tiegs, G. (2003) The flame retardants tetrabromobisphenol $\mathrm{A}$ and tetrabromobisphenol Abisallylether suppress the induction of interleukin-2 receptor alpha chain (CD25) in murine splenocytes. Toxicology, 184, 11-22.

9. Reistad, T., Mariussen, E. and Fonnum, F. (2005) The effect of a brominated flame retardant, tetrabromobisphenol-A, on free radical formation in human neutrophil granulocytes: the involvement of the MAP kinase pathway and protein kinase C. Toxicol. Sci., 83, 89-100.

10. Bruschi, F., Bianchi, C., Fornaro, M., Naccarato, G., Menicagli, M., Gomez-Morales, M.A., Pozio, E. and Pinto, B.
(2014) Matrix metalloproteinase (MMP)-2 and MMP-9 as inflammation markers of Trichinella spiralis and Trichinella pseudospiralis infections in mice. Parasite Immunol., 36, 540-549.

11. Jadhav, U., Chigurupati, S., Lakka, S.S. and Mohanam, S. (2004) Inhibition of matrix metalloproteinase-9 reduces in vitro invasion and angiogenesis in human microvascular endothelial cells. Int. J. Oncol., 25, 1407-1414.

12. Roy, R., Yang, J. and Moses, M.A. (2009) Matrix metalloproteinases as novel biomarkers and potential therapeutic targets in human cancer. J. Clin. Oncol., 27, 5287-5297.

13. Yu, W., Liu, J., Xiong, X., Ai, Y. and Wang, H. (2009) Expression of MMP9 and CD147 in invasive squamous cell carcinoma of the uterine cervix and their implication. Pathol. Res. Pract., 205, 709-715.

14. Scully, O.J., Bay, B.H., Yip, G. and Yu, Y. (2012) Breast cancer metastasis. Cancer Genomics Proteomics, 9, 311-320.

15. Blanckaert, V., Ulmann, L., Mimouni, V., Antol, J., Brancquart, L. and Chenais, B. (2010) Docosahexaenoic acid intake decreases proliferation, increases apoptosis and decreases the invasive potential of the human breast carcinoma cell line MDA-MB-231. Int. J. Oncol., 36, 737-742.

16. McGowan, P.M. and Duffy, M.J. (2008) Matrix metalloproteinase expression and outcome in patients with breast cancer: analysis of a published database. Ann. Oncol., 19, 15661572 .

17. Genersch, E., Hayess, K., Neuenfeld, Y. and Haller, H. (2000) Sustained ERK phosphorylation is necessary but not sufficient for MMP-9 regulation in endothelial cells: involvement of Ras-dependent and -independent pathways. J. Cell Sci., 113 Pt 23, 4319-4330.

18. Hong, S., Park, K.K., Magae, J., Ando, K., Lee, T.S., Kwon, T.K., Kwak, J.Y., Kim, C.H. and Chang, Y.C. (2005) Ascochlorin inhibits matrix metalloproteinase- 9 expression by suppressing activator protein-1-mediated gene expression through the ERK1/2 signaling pathway: inhibitory effects of ascochlorin on the invasion of renal carcinoma cells. J. Biol. Chem., 280, 25202-25209.

19. Mook, O.R., Frederiks, W.M. and Van Noorden, C.J. (2004) The role of gelatinases in colorectal cancer progression and metastasis. Biochim. Biophys. Acta, 1705, 69-89.

20. Garg, A. and Aggarwal, B.B. (2002) Nuclear transcription factor-kappaB as a target for cancer drug development. Leukemia, 16, 1053-1068.

21. Maraldi, T., Angeloni, C., Giannoni, E. and Sell, C. (2015) Reactive oxygen species in stem cells. Oxid. Med. Cell. Longev., 2015, 159080.

22. Panday, A., Sahoo, M.K., Osorio, D. and Batra, S. (2015) NADPH oxidases: an overview from structure to innate immunity-associated pathologies. Cell. Mol. Immunol., 12, $5-23$.

23. Cai, H. and Harrison, D.G. (2000) Endothelial dysfunction in cardiovascular diseases: the role of oxidant stress. Circ. Res., 87, 840-844.

24. Brown, D.I. and Griendling, K.K. (2009) Nox proteins in signal transduction. Free Radic. Biol. Med., 47, 1239-1253.

25. Giles, G.I. (2006) The redox regulation of thiol dependent signaling pathways in cancer. Curr. Pharm. Des., 12, $4427-$ 4443. 
26. Manea, S.A., Constantin, A., Manda, G., Sasson, S. and Manea, A. (2015) Regulation of Nox enzymes expression in vascular pathophysiology: Focusing on transcription factors and epigenetic mechanisms. Redox. Biol., 5, 358-366.

27. Morgan, M.J. and Liu, Z.G. (2011) Crosstalk of reactive oxygen species and NF-kappaB signaling. Cell Res., 21, 103-115.

28. Reddy, S.A., Huang, J.H. and Liao, W.S. (2000) Phosphatidylinositol 3-kinase as a mediator of TNF-induced NFkappa B activation. J. Immunol., 164, 1355-1363.

29. Woo, J.H., Lim, J.H., Kim, Y.H., Suh, S.I., Min, D.S., Chang, J.S., Lee, Y.H., Park, J.W. and Kwon, T.K. (2004) Resveratrol inhibits phorbol myristate acetate-induced matrix metalloproteinase-9 expression by inhibiting JNK and PKC delta signal transduction. Oncogene, 23, 1845-1853.

30. Jiang, F., Zhang, Y. and Dusting, G.J. (2011) NADPH oxidasemediated redox signaling: roles in cellular stress response, stress tolerance, and tissue repair. Pharmacol. Rev., 63, 218242.

31. Han, E.H., Park, J.H., Kang, K.W., Jeong, T.C., Kim, H.S. and Jeong, H.G. (2009) Risk assessment of tetrabromobisphenol A on cyclooxygenase-2 expression via MAP kinase/ NF-kappaB/AP-1 signaling pathways in murine macrophages. J. Toxicol. Environ. Health A, 72, 1431-1438.

32. Perkins, N.D. (2007) Integrating cell-signalling pathways with NF-kappaB and IKK function. Nat. Rev. Mol. Cell Biol., 8, 49-62.

33. Sato, T., Koike, L., Miyata, Y., Hirata, M., Mimaki, Y.,
Sashida, Y., Yano, M. and Ito, A. (2002) Inhibition of activator protein-1 binding activity and phosphatidylinositol 3kinase pathway by nobiletin, a polymethoxy flavonoid, results in augmentation of tissue inhibitor of metalloproteinases-1 production and suppression of production of matrix metalloproteinases-1 and -9 in human fibrosarcoma HT-1080 cells. Cancer Res., 62, 1025-1029.

34. Cho, H.J., Kang, J.H., Kwak, J.Y., Lee, T.S., Lee, I.S., Park, N.G., Nakajima, H., Magae, J. and Chang, Y.C. (2007) Ascofuranone suppresses PMA-mediated matrix metalloproteinase-9 gene activation through the Ras/Raf/MEK/ERK- and Ap1-dependent mechanisms. Carcinogenesis, 28, 1104-1110.

35. Karin, M., Liu, Z. and Zandi, E. (1997) AP-1 function and regulation. Curr. Opin. Cell Biol., 9, 240-246.

36. Chang, Y.C., Li, P.C., Chen, B.C., Chang, M.S., Wang, J.L., Chiu, W.T. and Lin, C.H. (2006) Lipoteichoic acid-induced nitric oxide synthase expression in RAW 264.7 macrophages is mediated by cyclooxygenase-2, prostaglandin E2, protein kinase A, p38 MAPK, and nuclear factor-kappaB pathways. Cell. Signal., 18, 1235-1243.

37. Fonnum, F., Mariussen, E. and Reistad, T. (2006) Molecular mechanisms involved in the toxic effects of polychlorinated biphenyls (PCBs) and brominated flame retardants (BFRs). J. Toxicol. Environ. Health A, 69, 21-35.

38. Reistad, T., Mariussen, E., Ring, A. and Fonnum, F. (2007) In vitro toxicity of tetrabromobisphenol-A on cerebellar granule cells: cell death, free radical formation, calcium influx and extracellular glutamate. Toxicol. Sci., 96, 268-278. 